\title{
Line identifications and intensities for the optical spectrum of RR Telescopii between 3180 and $9455 \AA$
}

\author{
F.L. Crawford ${ }^{1}$, F.C. McKenna ${ }^{1}$, F.P. Keenan ${ }^{1}$, L.H. Aller $^{2}$, W.A. Feibelman ${ }^{3}$, and S.G. Ryan ${ }^{4, \star}$ \\ 1 Department of Pure and Applied Physics, The Queen's University of Belfast, Belfast BT7 1NN, Northern Ireland, UK \\ e-mail: fergal.crawford@qub.ac.uk, f.mckenna@qub.ac.uk, f.keenan@qub.ac.uk \\ 2 University of California, Los Angeles, CA 90024, U.S.A. \\ e-mail: aller@bonnie.astro.ucla.edu \\ 3 NASA Goddard Space Flight Center, Greenbelt, MD 20771, U.S.A. \\ e-mail: feibelman@iue.gsfc.nasa.gov \\ 4 Anglo-Australian Observatory, PO Box 296, Epping, NSW 2121, Australia \\ e-mail: s.g.ryan@open.ac.uk
}

Received May 17; accepted July 1, 1999

\begin{abstract}
The symbiotic nova RR Telescopii has been observed with the $3.9 \mathrm{~m}$ telescope at the Anglo-Australian Observatory (AAO), using the University College London Echelle Spectrograph (UCLES) in conjunction with a Tek CCD. It displays a rich emission line spectrum, ranging in excitation from $\mathrm{N}_{\mathrm{I}}$ to [Ni VIII]. We present a list of 811 measured lines, with their suggested identifications and absolute line intensities, covering a wavelength range from $3180 \AA$ to $9455 \AA^{1}$. The absolute line intensities have been derived by comparing the high resolution data with a flux-calibrated low resolution spectrum taken with the Australian National University $2.3 \mathrm{~m}$ telescope. All of the lines have been successfully identified. Comparing our results with those of previous studies indicates that the $\mathrm{RR}$ Tel system is advancing towards higher degrees of ionisation.
\end{abstract}

Key words: binaries: symbiotic — line: identifications stars: individual (RR Tel)

\footnotetext{
Send offprint requests to: F.L. Crawford

* Present address: Physics Department, The Open University, Walton Hall, Milton Keynes MK7 6AA, UK.

1 Tables 2 and 3 are only available in electronic form at the CDS via anonymous ftp to cdsarc.u-strasbg.fr (130.79.128.5) or via http://cdsweb.u-strasbg.fr/Abstract.html
}

\section{Introduction}

The symbiotic nova RR Telescopii is one of a subclass of symbiotic stars. These are interacting binaries whose spectra indicate the presence of a late type star and a hot companion (Mürset et al. 1991). RR Tel has been the subject of many extensive spectroscopic investigations (see, for example, Aller et al. 1973) due to the great wealth of lines emitted since its outburst in 1944. Thackeray (1977) has compiled the observations of RR Tel from its outburst until the mid 1970s, and cites most relevant references.

McKenna et al. (1997) have presented a detailed calibrated list of 491 lines in the wavelength range $3444-$ $9229 \AA$ in RR Tel, at a resolution of $\sim 12 \mathrm{~km} \mathrm{~s}^{-1}$ (FWHM). However, it was impossible to distinguish many of the important spectral features due to blending at this resolution, and hence we have decided to further examine RR Tel's optical spectrum with a more powerful telescope. In this study, we have obtained new observations using a CCD detector with the University College London Echelle Spectrograph (UCLES) on the $3.9 \mathrm{~m}$ telescope at the Anglo-Australian Observatory (AAO). These data are of higher spectral resolution $\left(\sim 6 \mathrm{~km} \mathrm{~s}^{-1}\right)$ than the previous spectra, and so we are able to measure reliable emission line intensities for weaker features than was previously possible. The result is an improved line list for the optical spectrum of RR Tel with identifications and intensities of hitherto unidentified lines.

\section{Observations and data reduction}

Spectroscopic observations of RR Tel were obtained using the $3.9 \mathrm{~m}$ telescope at the Anglo-Australian Observatory 


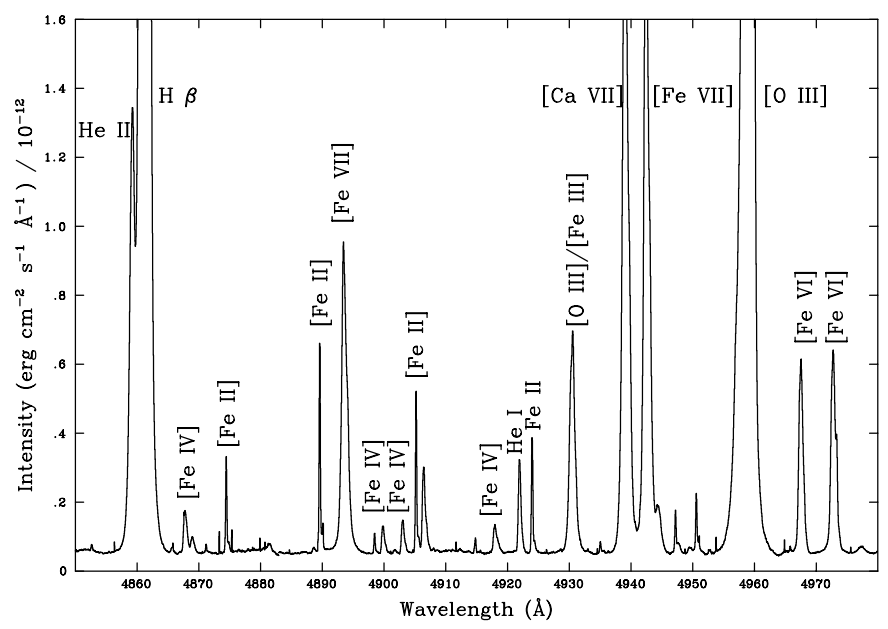

Fig. 1. The flux-calibrated high resolution optical spectrum of $\mathrm{RR}$ Tel in the wavelength range $4850-4980 \AA$, where the flux is in units of $10^{-12} \mathrm{ergs} \mathrm{cm}^{-2} \mathrm{~s}^{-1} \AA^{-1}$, featuring lines of the highly ionised species [Fe VII] at $4893.90 \AA$ and $4942.30 \AA$ and [O III] at $4931.00 \AA$ and $4958.91 \AA$, as well as the strong $\mathrm{H} \beta$ line at $4861.33 \AA$

on July 22 1996. The University College London Echelle Spectrograph was employed with the 31 lines $\mathrm{mm}^{-1}$ grating and $700 \mathrm{~mm}$ camera, along with the Tek CCD as a detector. The result was a high resolution, high signalto-noise $(\mathrm{S} / \mathrm{N})$ spectrum of $\mathrm{RR}$ Tel over the near-UV, optical and near-IR regions, $3100-9800 \AA$. The resolution was $\lambda / \Delta \lambda \simeq 50000$, a factor of 2 greater than for the previous data from the Cerro Tololo Inter-American Observatory (CTIO) (McKenna et al. 1997), and the S/N was $\simeq 20$ pixel $^{-1}$ in the continuum, similar to the CTIO observations. This allowed a greater dynamic range than with earlier observations, and enabled us to measure reliable line intensities for much weaker features than was previously possible. A section of our high resolution data is shown in Fig. 1.

In order to absolutely flux-calibrate our data, it was necessary to obtain low resolution spectra of RR Tel. These were obtained on August 21996 with the Australian National University $2.3 \mathrm{~m}$ telescope and double beam spectrograph, which gave a resolution of $\simeq 2 \AA$ pixel $^{-1}$. However, this instrumental setup only gave useful data for wavelengths in the range $3490 \leq \lambda \leq 5530 \AA$. Line fluxes for wavelengths outside this range were derived from the He II recombination line spectrum (see Sect. 4). The low resolution spectrum is shown in Fig. 2, where, due to seeing of $\sim 2^{\prime \prime}$, the accuracy of the flux calibration is estimated at $\pm 10 \%$.

All CCD images were reduced using the Image Reduction and Analysis Facility (IRAF) version 2.3 implemented at the Queen's University, Belfast STARLINK node. Standard procedures were followed in the reduction of the images to one dimensional spectra. Low resolution spectra were optimally extracted and flux-calibrated using standards from AAO lists. The densely-packed

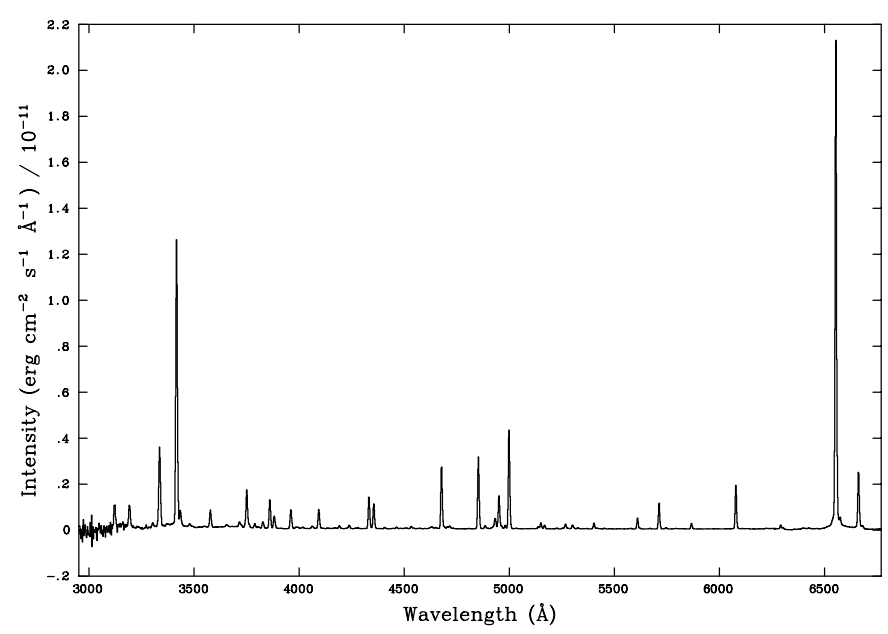

Fig. 2. The flux-calibrated low resolution optical spectrum of RR Tel, where the flux is in units of $10^{-11} \operatorname{ergs~cm}^{-2} \mathrm{~s}^{-1} \AA^{-1}$

Table 1. An illustration of the changes occurring in the intensities of lines as RR Tel progresses towards a higher degree of ionisation. All intensities are given relative to $\mathrm{H} \beta=100$

\begin{tabular}{lllll}
\hline \hline Ion (wavelength) & I.P. (eV) & $I(1968)$ & $I(1993)$ & $I(1996)$ \\
\hline Fe II (3779.58) & 7.9 & 0.170 & 0.212 & 0.088 \\
Fe II (3824.91) & 7.9 & 0.594 & 0.480 & 0.265 \\
Fe II (3914.48) & 7.9 & 0.453 & 0.184 & 0.078 \\
Si II (3856.02) & 8.2 & 0.453 & 0.324 & 0.170 \\
Si II (3862.59) & 8.2 & 0.665 & 0.548 & 0.265 \\
He II (3923.48) & 24.6 & 0.764 & 1.164 & 1.139 \\
[Fe IV] (4206.30) & 30.7 & 0.363 & 0.232 & 0.308 \\
[Ne III] (3868.74) & 41.0 & 60.83 & 57.20 & 75.38 \\
[Fe V] (3895.70) & 54.8 & 2.122 & 2.552 & 2.678 \\
[Ne IV] (4724.15) & 63.5 & 5.374 & 3.032 & 3.378 \\
[Fe VII] (4942.30) & 100.0 & 6.046 & 6.240 & 8.092 \\
[Ca VII] (4940.30) & 108.8 & - & 6.040 & 10.03 \\
\hline \hline
\end{tabular}

échelle spectra were extracted using cosmic ray rejection algorithms for sky regions only, as cosmic ray hits in the spectra themselves could be confused with real emission features. Multiple high resolution échelle images enabled us to median filter the extracted spectra to subsequently eliminate cosmic rays. Once reduced to one dimensional spectra, the data were input to the user-friendly spectrum analysis program DIPSO (Howarth et al. 1996) for further analysis.

\section{Line identifications}

The velocity-corrected measurement for each line of our RR Tel spectrum is listed, in angstroms, in Col. (1) of Table 2. In Col. (5) the suggested identities of the major contributors to these lines are listed, where the ion is followed by the laboratory wavelength. A superscript at one of these wavelengths refers to a note at the end of the table. 

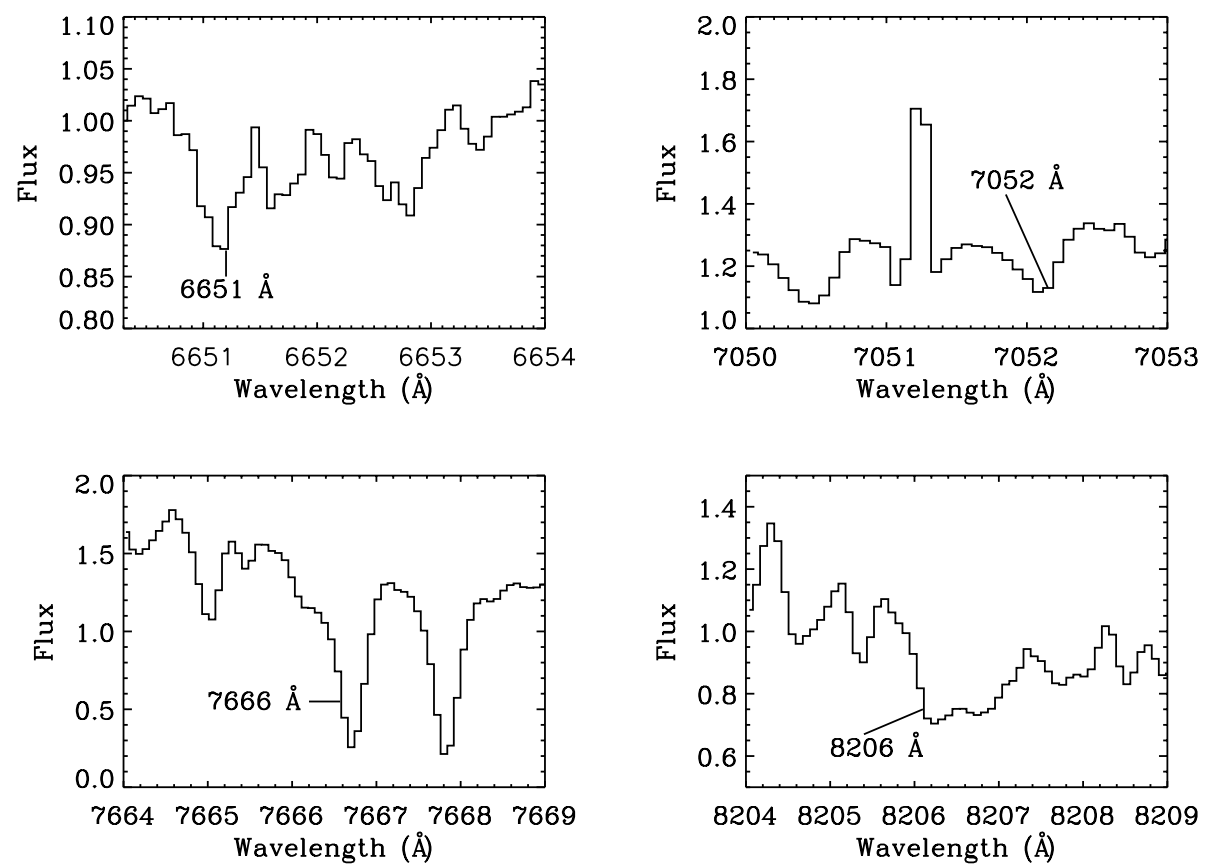

Fig. 3. Titanium oxide absorption bands detected in the spectrum of RR Tel at $6651 \AA$, $7052 \AA, 7666 \AA$ and $8206 \AA$

The methods employed in identifying the many emission lines were the same as those used by McKenna et al. (1997), and are described therein. However, several of their listed identifications were found to be erroneous; our greater spectral resolution allowed us to discard some of their lines, and to re-identify others. One finding list not used by McKenna et al. which was employed in the present study is the near-UV and optical line list of van Hoof (1998). However, it is still apparent that not all of the lines in this study are fully resolved. For example, the line at $\lambda 3724.99 \AA$ appears to be a blend of Mn II $\lambda 3724.81 \AA$, O II $\lambda 3725.30 \AA$ and [O II] $\lambda 3726.08 \AA$.

The line widths and shapes were also considered in identifications - most lines of a given ion and multiplet were of similar width and shape. Also, the presence of several lines from a given multiplet makes it likely that other lines from that multiplet will be present. Furthermore, lines were wider for the higher stages of ionisation; for example, the average velocity width for the Fe II and [Fe II] lines was about $\overline{\Delta v}=14 \mathrm{~km} \mathrm{~s}^{-1}$; the average width for [Fev] was $41 \mathrm{~km} \mathrm{~s}^{-1}$, and an average width of $62 \mathrm{~km} \mathrm{~s}^{-1}$ was found for the [Fe VII] lines. Similarly, O II lines were found to have an average velocity width of $30 \mathrm{~km} \mathrm{~s}^{-1}$ and the average width of O IV lines was found to be $44 \mathrm{~km} \mathrm{~s}^{-1}$. We notice the similarity in velocity widths of the $[\mathrm{FeV}]$ $\left(\overline{\Delta v}=41 \mathrm{~km} \mathrm{~s}^{-1}\right)$ and O IV $\left(\overline{\Delta v}=44 \mathrm{~km} \mathrm{~s}^{-1}\right)$ lines, where the ionisation potentials are $56 \mathrm{eV}$ and $54.93 \mathrm{eV}$, respectively.

In cases where it was unclear which of a number of possible ions were contributing to lines, the average velocity width for each ion was compared to the width of the line in question. For example, the line at $\lambda 3668.99 \AA$ was thought to be a blend of H I $\lambda 3669.46 \AA$ and [Ca VI] $\lambda 3669.10 \AA$. The measured line was significantly broader $\left(\Delta v=62 \mathrm{~km} \mathrm{~s}^{-1}\right)$ than the other $\mathrm{H}$ I (multiplet 5) lines which had an average velocity width $\overline{\Delta v}=50 \mathrm{~km} \mathrm{~s}^{-1}$, so [Ca VI] is listed as a possible co-contributor. On the other hand, H I (multiplet 3) lines were found to have an average velocity width $\overline{\Delta v}=53 \mathrm{~km} \mathrm{~s}^{-1}$, and the line at $\lambda 3721.51 \AA$ agreed well with this, so the listing of [S III] at $\lambda 3721.70 \AA$ by McKenna et al. (1997) was discarded. In all cases where blends are listed, the contributors are listed in decreasing order of perceived importance.

It is possible that some $\mathrm{O}$ VI emission has been detected at $\lambda \lambda 3811.36$ and $3834.24 \AA$. The $3811.36 \AA$ line was listed by McKenna et al. (1997), although its doublet partner at $3834.24 \AA$ was not. Attempts were made to ascertain whether or not these lines are real, as both features are blends, by comparing the profiles of the $\mathrm{O}$ III (multiplet 2) lines at $\lambda \lambda 3759.87,3774.00$ and $3791.26 \AA$ to that of the line at $3811.21 \AA$, which we have identified as a blend of O III $\lambda 3810.96 \AA$ and O VI $\lambda 3811.36 \AA$. However, this method proved inconclusive.

There is evidence of O VI emission at the red end of the spectrum. We find extremely broadened features at $\lambda \lambda 6825$ and $7082 \AA$, which have been identified by Espey et al. (1995) as the Raman-scattered ultraviolet O VI $\lambda \lambda 1032$ and $1038 \AA$ resonance lines (Schmid 1989). These lines have split profiles, probably due to relative gas motions in the red giant's wind.

The line listed in Table 2 at $4446.29 \AA$ is thought to be a blend of Fe II $\lambda 4446.25 \AA$ and [Ni VIII] $\lambda 4446.20$. The [Ni VIII] line is important as it has the highest ionisation potential (I.P. $\sim 136 \mathrm{eV}$ ) of all the lines measured in this 
study. It proved impossible to resolve the [Ni VIII] totally for the table, but the line width can be roughly measured as $\Delta v \simeq 29 \mathrm{~km} \mathrm{~s}^{-1}$.

Thackeray (1977) noted that the presence of the late type star in the RR Tel system is confirmed by the detection of $\mathrm{TiO}$ bandheads in the near infrared and red regions of the spectrum. These features are known to be prominent in mid M type stars (Kirkpatrick et al. 1991; Vardya 1992) and we have made definitive identifications of the $\mathrm{TiO}$ absorption bands (see Fig. 3).

\section{Line intensities}

The measured intensity of each line (in $\mathrm{erg} \mathrm{cm}^{-2} \mathrm{~s}^{-1}$ ) is listed in Col. (3) of Table 2. These intensities were obtained over the wavelength range $3490 \leq \lambda \leq 5530 \AA$ using the methods described in McKenna et al. (1997). The error in each line intensity listed in Table 2 is estimated at $<15 \%$, except where indicated by the footnotes. Outside the range $3490 \leq \lambda \leq 5530 \AA$, the intensities were measured using recombination line intensity ratios in He II (Hummer \& Storey 1987; Storey \& Hummer 1995). Ionised helium lines at $\lambda \lambda 3203.16,5952.96,5977.05,6036.81,6074.22$, $6170.72,6233.84,6406.41,6527.12$ and $6683.22 \AA$ were compared to the $n=4 \rightarrow 3$ transition at $4685.68 \AA$. However, the intensities that this method yields are not as accurate as those found using the low resolution spectrum, and hence errors of $\sim 20 \%$ in the intensities of the lines listed in Table 3 are expected.

The line intensities were corrected for interstellar extinction (see Col. (4) in Table 2) using the coefficients listed in Cardelli et al. (1989) for the $U, B, V$ and $R$ spectral regions. $E(B-V)$ for $\mathrm{RR}$ Tel is well quantified as equal to 0.08 (Jordan et al. 1994).

McKenna et al. (1997) were the first to produce an absolutely calibrated line list of the optical spectrum of RR Tel. However, Aller et al. (1973) list line intensities relative to $\mathrm{H} \beta$. Thus, it was possible to carry out an approximate comparision between the intensity of RR Tel's lines in 1968 (Aller et al. 1973), 1993 (McKenna et al. 1997) and 1996 , by scaling the line intensities in this study relative to $\mathrm{H} \beta$. It was found that the strengths of the lines for low ionisation species (for example, He I, [O I], Fe II and $\mathrm{O}$ III) are decreasing, while the line intensities of species of higher degrees of ionisation (for example, [Fe VII]) are increasing (see Table 1). This is the expected result, as RR Tel's emission spectrum has been well documented as advancing towards higher excitation (Kenyon et al. 1993; Allen 1980).

\section{Component structure in the nebular lines}

The data used in this investigation are of sufficiently high resolution to show component structure in many of the

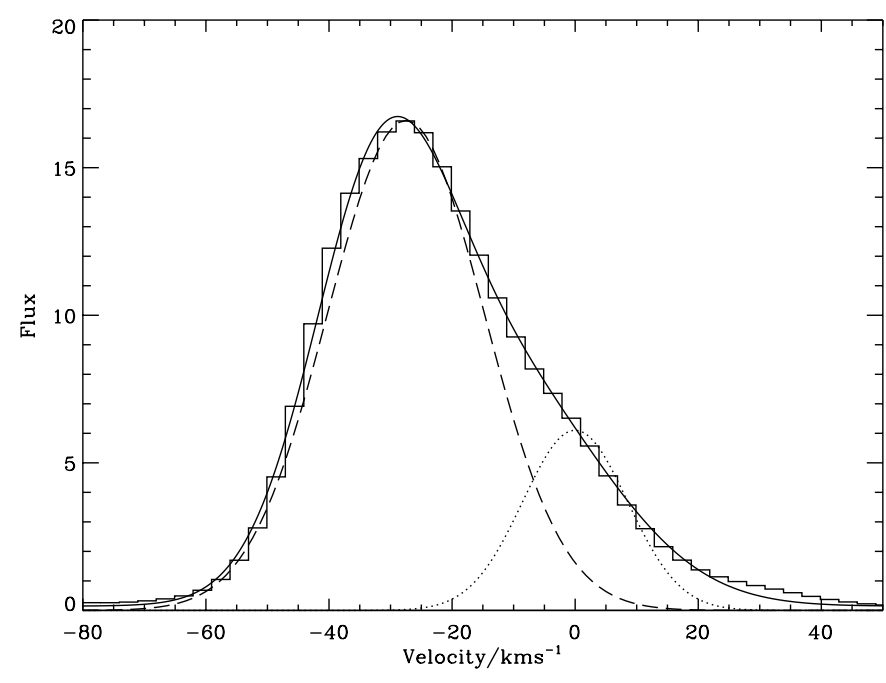

Fig. 4. The [O III] $4363 \AA$ emission line profile, in RR Tel (flux measured in arbitrary units). The line profile has been fitted using two components, separated by $\sim 28 \mathrm{~km} \mathrm{~s}^{-1}$, and indicated by dashed and dotted lines, while the solid line shows the approximate sum of these

nebular lines of RR Tel. For example, the [O III] $\lambda 4363 \AA$ line shows definite evidence of a two component structure in the nebular emission (see Fig. 4). Schild \& Schmid (1996) note that this may illustrate a combination of a high density component with a logarithmic electron density $\left(N_{\mathrm{e}}\right.$ in $\left.\mathrm{cm}^{-3}\right)$ of $\sim 8$, along with a low density component $\left(\log N_{\mathrm{e}} \sim 5.5\right)$, which we found to be blueshifted by $\sim 28 \mathrm{~km} \mathrm{~s}^{-1}$ with respect to the high density feature (see Fig. 4), in comparison to the blueshift of $\sim 20 \mathrm{~km} \mathrm{~s}^{-1}$ found by Schild \& Schmid.

The [O III] lines at $\lambda \lambda 4959$ and $5007 \AA$ also show component structure. However, in these cases, the line profiles comprise at least three components, making the modelling process much more complicated (see Fig. 5). A detailed analysis of these lines may be used to demonstrate additional physical characteristics of the RR Tel system, such as its winds (Nussbaumer \& Dumm 1997). Similar structural attributes to those of the [O III] lines may also be found in the profiles of O II, N II, N III and Ne IV. In the future we intend to pursue a detailed analysis of these and all the other nebular line profiles, in order to build up a comprehensive picture of the component structure of the RR Tel system.

We also find very strong component structure in the features at $\lambda \lambda 6825$ and $7082 \AA$. These are believed to be Raman-scattered O vi emission from the $\lambda \lambda 1032$ and $1038 \AA$ resonance lines (Espey et al. 1995; Schmid 1989). The two components of both features are separated by $\sim 330 \mathrm{~km} \mathrm{~s}^{-1}$. 


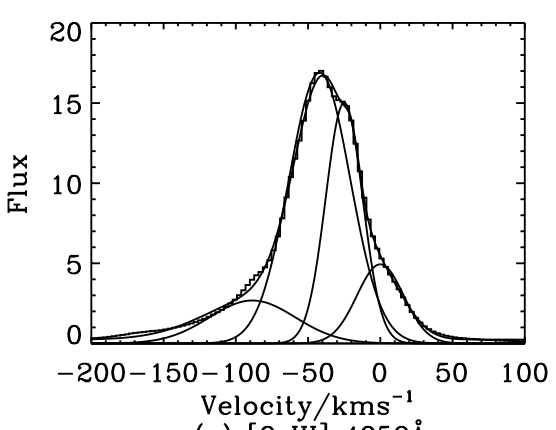

(a) [O III] $4959 \AA$

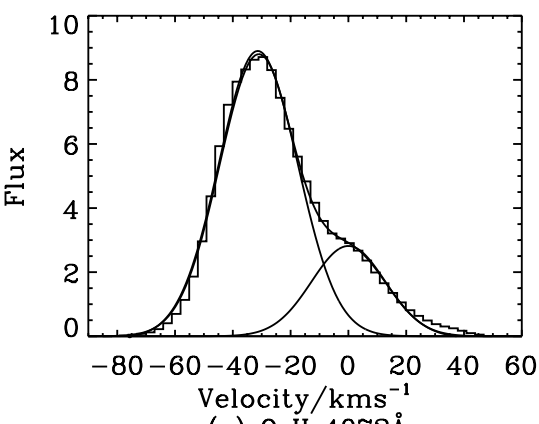

(c) 0 II $4072 \AA$

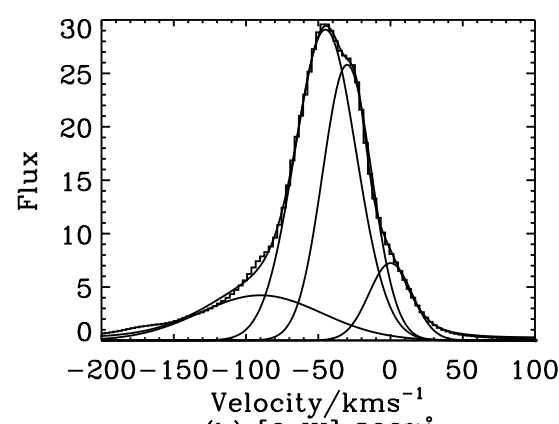

(b) $[0$ III $] 5007 \AA$

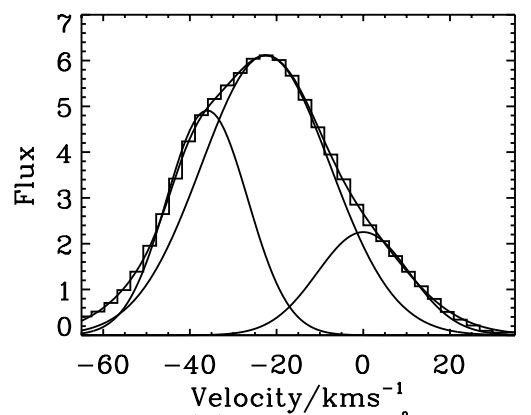

(d) Ne IV $4714 \AA$

Fig. 5. The [O III] line profiles at a) $4959 \AA$ and b) $5007 \AA$, showing four-component structure; c) O II $\lambda 4072 \AA$ showing two component structure; d) three-component structure in Ne IV $\lambda 4714 \AA$ (flux measured in arbitrary units)

\section{Conclusion}

We present a detailed calibrated line list of the optical spectrum of RR Tel, listing 811 lines with identifications, including 320 lines not listed by McKenna et al. (1997). It was also necessary to discard some of the lines listed in the McKenna et al. study and to re-identify others; this was made possible by the improved resolution of the present study.

In this study, we have further investigated the changes that are continually occurring in the emission spectrum of RR Tel. We have found that the tendency towards higher levels of ionisation has continued (see Table 1), as noted by Kenyon et al. (1993) and Allen (1980).

We have noted strong component structure in many of the nebular emission lines. This structure can involve 2 or more components and may be evidence of a combination of high $\left(\log N_{\mathrm{e}} \sim 8\right)$ and low density $\left(\log N_{\mathrm{e}} \sim 5.5\right)$ emitting zones in the plasma (Schild \& Schmid 1996).

$\mathrm{TiO}$ absorption bandheads were found in the near infrared and red regions of the spectrum, which indicate the presence of the late type star in the RR Tel system, as noted by Thackeray (1977). These features are prominent in mid M type stars (Kirkpatrick et al. 1991). Figure 3 shows our identifications of these bands.

This optical line list compliments the earlier work of Zuccolo et al. (1997) who have presented a study of the changes in the ultraviolet spectrum of RR Tel between 1978 and 1993, using the International Ultraviolet Explorer (IUE) satellite.
Acknowledgements. FLC is grateful to the Department of Education for Northern Ireland for the award of a research studentship. FCM is grateful to PPARC for her postdoctoral funding. This work was supported by NATO travel grant CRG/930722 and the National Science Foundation through Grants Nos. AST 90-14133, AST 93-13991, and AST 94-16985 to the University of California, Los Angeles. We are grateful to Alan Fitzsimmons, QUB, for obtaining the observations presented in this paper. We are also especially grateful to the referee, Regina Schulte-Ladbeck, for her invaluable criticisms and suggestions. This research has made use of NASA's Astrophysics Data System Abstract Service.

\section{References}

Allen D.A., 1980, MNRAS 192, 521

Aller L.H., Polidan R.S., Rhodes E.J., Wares G.W., 1973, Ap\&SS 20, 93

Cardelli J.A., Clayton G.C., Mathis J.C., 1989, ApJ 345, 245

Espey B.R., Schulte-Ladbeck R.E., Kriss G., et al., 1995, ApJ 454, L61

Howarth I.D., Murray J., Mills D., Berry D.S., 1996, DIPSO (V3.4), Starlink User Note, No. 50.20

Hummer D.G., Storey P.J., 1987, MNRAS 224, 801

Jordan S., Mürset U., Werner K., 1994, A\&A 283, 475

Kenyon S.J., Mikoljewska J., Mikoljewska M., Polidan R.S., Slovak M.H., 1993, AJ 106, 1573

Kirkpatrick J.D., Henry T.J., McCarthy D.W., 1991, ApJS 77, 417

McKenna F.C., Keenan F.P., Hambly N.C., et al., 1997, ApJS 109, 225

Mürset U., Nussbaumer H., Schmid H.M., Vogel M., 1991, A\&A 248, 458 
Nussbaumer H., Dumm T., 1997, A\&A 323, 387

Schild H., Schmid H.M., 1996, Nebular Density Profiles of PU Vul and RR Tel, in Mikolajewska J. (ed.) Physical Processes in Symbiotic Binaries and Related Systems Schmid H.M., 1989, A\&A 211, L31

Storey P.J., Hummer D.G., 1995, MNRAS 272, 41

Thackeray A.D., 1977, Mem. R. Astron. Soc. 83, 1 van Hoof P.A.M., 1998, A Comprehensive Line List for Atomic Transitions on the WWW, in Mezzacappa A. (ed.) Stellar Evolution, Stellar Explosions, and Galactic Chemical Evolution. IOP Publishing, Bristol (in press)

Vardya M.S., 1992, Long Period Variable Stars, in Maran S.P. (ed.) The Astronomy and Astrophysics Encyclopedia. Cambridge University Press, Cambridge

Zuccolo R., Selvelli P., Hack M., 1997, A\&AS 124, 425 